\section{The Bristol cataract listing survey: Profile of listed patients with visual acuity $6 / 12$ or better}

\begin{abstract}
Introduction The aim of this study was to assess the vision-related quality of life (VR-QOL) in patients with good distance Snellen visual acuity (VA) who are listed for cataract surgery. Methods An observational cross-sectional prospective study of patients listed for cataract surgery. VA and VR-QOL data using the VCMI questionnaire were collected on patients attending preoperative assessment during June 2002.

Results A total of 397 cataract patients were listed during this month. Following exclusions there were 378 eligible individuals, $210(56 \%)$ of whom had a VA of $6 / 12$ or better in the eye scheduled for surgery. Of these, $40 \%$ patients had only mild VR-QOL impairment. More than half of the patients with good VA (6/12 or better) in the surgery eye and mild VR-QOL impairment described their vision as poor in this eye. However, most of these patients were not dissatisfied with their overall level of vision. Conclusion A significant number of patients listed for cataract surgery with VA of 6/12 or better had only mild VR-QOL impairment and were not dissatisfied with their overall level of vision. The decision to list a patient for surgery may have been based on the patient's perception of monocular vision rather than their quality of life.

Eye (2005) 19, 406-410. doi:10.1038/sj.eye.6701511

Published online 6 August 2004
\end{abstract}

Keywords: listing for cataract surgery; visionrelated quality of life (VR-QOL); visual acuity $6 / 12$ or better; thresholds for surgery

2003

Accepted: 9 February 2004 Published online: 6 August 2004

Proprietary interests: None Research Funding: None

\section{Introduction}

In the clinical setting, monocular visual acuity (VA) is used as a primary tool for judging the need for cataract surgery. However, in the UK
R Malik , NA Frost² ${ }^{2}$ JM Sparrow ${ }^{1}$ and L Hale ${ }^{3}$

National Cataract Survey of 1998, 33\% of patients who were listed for cataract surgery had a visual acuity of $6 / 12$ or better. ${ }^{1}$ It is well recognised that visual impairment secondary to cataract cannot be adequately described in terms of a single loss of visual function. ${ }^{2}$ In some instances, (high contrast) VA can be reasonably well preserved in the presence of symptomatic visually impairing cataract, but most British ophthalmologists use other visual tests infrequently. ${ }^{3}$ Nor is there widespread familiarity with quality of life instruments for cataract assessment. ${ }^{4}$ In the absence of a formal assessment of other aspects of vision and visual quality of life, the functional status of many cataract surgery patients with good acuity remains poorly understood. Given that individuals with good visual function and fewer symptoms are less likely to benefit from cataract surgery, ${ }^{5}$ it is important to investigate the visual and functional status of cataract patients with good VA in more detail. The aim of this study was to assess the vision-related quality of life (VR-QOL) in patients with good distance Snellen VA who are listed for cataract surgery.

\section{Method}

An observational cross-sectional prospective study of patients listed for cataract surgery was carried out at Bristol Eye Hospital in June 2002. Shortly after being listed for cataract surgery at Bristol Eye Hospital, all patients are seen in a Pre-Operative Assessment Clinic (POAC) for an ophthalmic examination, biometry, and to be given a date for surgery. VA and VR-QOL data were collected on all patients who attended POAC between 1 June 2002 and 31 June 2002 inclusive. Although we have only reported patients with VA 6/12 or better here, data were collected on all listed patients regardless of VA. These acuities were recorded during routine 
outpatient clinics and subsequently analysed for the purpose of this study. All VA measurements were performed by nursing staff who were unaware of the outcome measure of ' $6 / 12$ or better'.

All patients who were scheduled to attend during this month were sent a VR-QOL questionnaire (VCMI questionnaire) by post. The patients were asked to return the completed questionnaire at their clinic visit. Additional questionnaires were provided at POAC for patients who had not received or who had mislaid their postal questionnaire. Patients were encouraged to complete their questionnaires themselves. A note was made of any nonresponders to the questionnaire. The VCMI questionnaire is a valid and reliable measure of VR-QOL and contains 10 broadly applicable questions referring to physical, social, and psychological issues. ${ }^{6}$ The VCMI score ranges from 0.0 (no problem) to 5.0 (extreme problem) with 50 intervals, and is strongly associated with responses to questions about a wide range of quality of life issues including mobility, reading and leisure. VR-QOL impairment was classified as 'mild' (score 0-1.0), 'moderate' (1.1-2.0), or 'severe' (2.1-5.0). Patients were also asked about their overall satisfaction with vision and about their opinion of the quality of their vision in each eye. In addition, on attendance at POAC, a standard data collection sheet was completed by the nursing and medical staff. The age and sex of each patient were recorded. VA at listing was measured as the best measured acuity (pinhole or glasses, whichever better). Ocular comorbidity, the type of cataract in the listed eye, and the surgical status of the fellow eye were documented. Whenever cataract surgery was planned for nonvisual reasons, this was specifically recorded (eg interference with visual field interpretation, difficult retinal view, lens-induced glaucoma). In accordance with the national cataract survey, patients below the age of 50 years were excluded from the data set. A customised Microsoft Access database was used for data analysis. Univariable $\chi^{2}$ tests were used for examining group differences.

\section{Results}

A total of 397 patients were seen in POAC between 1 and 31 June 2002. In all 11 patients below the age of 50 years were excluded and eight patients were excluded because of surgery for nonvisual reasons. Of these, 3 patients were listed because of anisometropia, 2 patients had lens-induced glaucoma, 2 patients had a difficult retinal view, and one patient had visual fields that were difficult to interpret because of cataract. The majority of eligible listed patients were female $(261 / 378,69 \%)$. Age ranged from 50 to 94 years. The mean age was 77 years and median 78 years.

A total of 210 patients $(56 \%)$ had a VA of $6 / 12$ or better in the eye scheduled for surgery. The VCMI questionnaire response rate in this group was 93\% (195/ 210). The nonresponders were not significantly different from the responders in terms of operative eye (first or second), sex, or age distribution $(P=0.27,0.34$ and 0.60 , respectively $\chi^{2}$ test). The VAs in the better eye (for the categories $6 / 6$ or better, $6 / 9$, and $6 / 12$ ) of the responders and nonresponders in this group were also similar $(P=0.38)$. Table 1 compares the VA at listing with the UK National Cataract Survey 1998 and with data from one of our previous audits. The previous audit of 208 patients undertaken at Bristol Eye Hospital in 1999 showed that, at that time, listing VA was similar to national levels $\left(P=0.78, \chi^{2}=0.49\right.$, Table 1$)$. In our current sample, $56 \%$ of patients had a VA of 6/12 or better in the surgery eye compared to only $32 \%$ in the 1998 National Survey $\left(P=0.002, \chi^{2}=12.7\right)$. In all, $40 \%$ of the patients in our current sample who had VA of $6 / 12$ or better had a mild VR-QOL impairment (Table 2). Out of these patients who had VA of $6 / 12$ or better in the listed eye and only mild VR-QOL impairment, $96 \%$ had VA of $6 / 12$ or better in the other (nonlisted) eye (Table 3 ). A similar proportion had VA of 6/12 or better in their worse eye (listed or nonlisted, whichever worse), and all patients had acuity

Table 2 VR-QOL distribution in patients with a VA of 6/12 or better in the listed eye

\begin{tabular}{lc}
\hline VR-QOL impairment & Frequency, N (\%) \\
\hline Mild & $77(40)$ \\
Moderate & $59(30)$ \\
Severe & $59(30)$ \\
Total & $195(100)$ \\
\hline
\end{tabular}

Table 1 VA at listing compared with the National Cataract Survey 1998.

\begin{tabular}{lcrrr}
\hline & $6 / 12$ or better & $6 / 18-6 / 60$ & $<6 / 60$ & Total \\
\hline Nat. cat. survey 1998 (\%) & $5385(31)$ & $9390(54)$ & $2670(15)$ & $17445(100)$ \\
Bristol 1998/1999 (\%) & $62(30)$ & $120(58)$ & $26(12)$ & $208(100)$ \\
Bristol 2002 $(\%)$ & $210(56)$ & $134(35)$ & $34(9)$ & $378(100)$ \\
\hline
\end{tabular}

a'Listing VAs Bristol 2002 are significantly different from the National Survey $1998\left(P=0.002, \chi^{2}=12.7\right)$. 
Table 3 Patients with good VA (6/12 or better) and mild VR-QOL impairment: VA in other (nonlisted) eye, in better eye and worse eye

\begin{tabular}{lccc}
\hline$V A$ & & Frequency, $N(\%)$ & \\
\cline { 2 - 3 } & Other (non-listed) eye & Better eye & Worse eye \\
\hline $6 / 4$ & $4(5)$ & $4(5)$ & 0 \\
$6 / 5$ & $10(13)$ & $10(13)$ & $2(2)$ \\
$6 / 6$ & $19(25)$ & $21(27)$ & $6(8)$ \\
$6 / 9$ & $25(32)$ & $31(41)$ & $39(51)$ \\
$6 / 12$ & $16(21)$ & $11(14)$ & $27(35)$ \\
$6 / 18-6 / 60$ & $3(4)$ & 0 & $3(4)$ \\
$<6 / 60$ & 0 & 0 & 0 \\
Total & $77(100)$ & $77(100)$ & $77(100)$ \\
$6 / 12$ or better & $74(96)$ & $77(100)$ & $74(96)$ \\
\hline
\end{tabular}

Table 4 Patients with good VA (6/12 or better) in eye for surgery and mild VR-QOL impairment, how patients described their eyesight in the listed eye during the last month

\begin{tabular}{lc}
\hline Subjective eyesight in listed eye & Frequency, N (\%) \\
\hline Very good & $1(1)$ \\
Fairly good & $9(12)$ \\
Average & $19(25)$ \\
Fairly poor & $33(43)$ \\
Very poor & $10(13)$ \\
Cannot see anything & 0 \\
Not known & $5(6)$ \\
Total & $77(100)$ \\
\hline
\end{tabular}

of $6 / 12$ or better in their best eye. Of the 77 patients with VA of $6 / 12$ or better in the eye for surgery and mild VR-QOL impairment, 36 (47\%) were pseudophakic in the fellow eye. The type of cataract in the listed eye was variable. A total of $24(31 \%)$ had a predominantly nucleosclerotic cataract, $16(21 \%)$ had cortical cataract, 26 (34\%) had mixed cataract, and seven (9\%) had posterior subcapsular cataract (data on type of cataract were not available in $4 / 77$ patients). Despite good VA and VR-QOL, 33 (43\%) of these patients described their vision as 'fairly poor' in the listed eye and a further $10(13 \%)$ described it as 'very poor' (Table 4). However, most of them $(64,83 \%)$ were not dissatisfied with their overall level of vision (Table 5).

\section{Discussion}

Cataract surgery is one of the most common elective surgical procedures performed in the UK. ${ }^{7}$ A Swedish study estimated that the direct costs to society in 1 year caused by 1458 patients awaiting cataract surgery is equivalent to the cost of operating on 800 eyes. ${ }^{8}$ Meeting the high demand for cataract surgery remains a
Table 5 Patients with good VA (6/12 or better) in the surgery eye and mild VR-QOL impairment. How patients described their overall satisfaction with sight during the last month

\begin{tabular}{lc}
\hline Satisfaction with sight & Frequency, N (\%) \\
\hline Very satisfied & $7(9)$ \\
Fairly satisfied & $28(36)$ \\
Mixed feelings & $29(38)$ \\
Fairly dissatisfied & $4(5)$ \\
Very dissatisfied & $2(3)$ \\
Extremely dissatisfied & $1(1)$ \\
Not known & $6(8)$ \\
& \\
Total & $77(100)$ \\
\hline
\end{tabular}

challenge, and it is in the public interest to ensure that available cataract surgery capacity is used most appropriately. The issues of appropriateness and possible overutilisation of cataract surgery have been raised by several investigators. ${ }^{9-14,16,17}$ Damiano et al ${ }^{13}$ observed that $3 \%$ of pre operative cataract patients reported having no trouble with their vision. In Norway, Sletteberg et al ${ }^{11}$ found that one of every six patients listed for cataract surgery felt their visual problems to be so small that the operation was not presently needed. Alonso et al ${ }^{14}$ reported that the proportion of preoperative cataract patients with VF-14 (visual function) scores of 100 (ie no difficulty performing the specified activities) ranged from 3.3 to $5.6 \%$ in Manitoba (Canada), Denmark, Barcelona (Spain), and the United States. Some patients who report no functional impairment may have other cataract symptoms, ${ }^{15}$ but in the study of Alonso et al ${ }^{14}$ the proportions of preoperative patients who reported no trouble with their vision ranged from 2.0 to $4.6 \%$. A recent study in Canada of 1098 cataract surgeries found that only $18 \%$ of patients had a preoperative VA of $20 / 40$ $(6 / 12)$ or better in the affected eye. They found that there was not enough information to justify surgery in $8 \%$ of 
cases. ${ }^{16}$ Tobacman et al ${ }^{17}$ assessed the relationship between preoperative appropriateness of cataract surgery as rated by an expert panel against improvement in VA postoperatively. Appropriateness was rated by the RAND-UCLA method, based on objective measures from available literature and a mutual agreement between a panel of experts. Improvement in at least two Snellen chart lines occurred in $36 \%$ of patients who had been rated as 'inappropriate'. Data regarding the appropriateness of cataract surgery in the UK are scarce. Desai et $\mathrm{l}^{18}$ reported that $40 \%$ of preoperative patients indicated no dysfunction on the VR-SIP (a modification of the Sickness Impact Profile) questionnaire.

In the present study, the sample studied provides information exclusively on patients already listed for cataract surgery. The aim of this study was to document the profile of listed patients with surgical eye VA of $6 / 12$ or better. This subgroup of patients is of particular interest in the decision-making process when offering surgery. Small changes in threshold criteria can have a large impact on the number of patients eligible for surgery. A detailed understanding of this area is therefore important for healthcare practitioners who are involved with listing patients for cataract surgery. The sample studied here illustrates adequately that there is a range of severity of quality of life impairment, and that there is no simple threshold in terms of symptoms for listing patients.

The proportion of patients in this group was significantly higher in our study compared with national data of 1998, and our own data from 1998/99 (Table 1). This change may demonstrate more generous thresholds for surgery in our department or different population characteristics in our Bristol sample. However, as it has been 5 years since the national data collection, the difference may well reflect a national change in surgical thresholds. If the aim of cataract surgery is to improve VR-QOL, there is likely to be a level beyond which the postoperative improvement of VR-QOL is minimal and counterbalanced by the risks and intrinsic limitations of the operation. In our study, a high proportion of patients with relatively good surgical eye acuity had only mild VR-QOL impairment, and so the reasons for surgery need to be questioned. Many of the patients had relatively good quality of life, fairly good vision in both eyes, and were reasonably satisfied with their vision. VR-QOL is known to depend on vision in both eyes and is influenced more strongly by the level of vision in the better eye, which may explain the discordance between monocular perceptions of vision and VR-QOL observed in our study.

Although a high proportion of patients with good VA and mild VR-QOL impairment were satisfied with their vision overall, they generally described vision in the affected eye as poor. Many of the patients with poor vision in one eye and good quality of life may have been expecting to be offered surgery, and may have been referred by their optometrist with such an expectation. The clinicians' judgments in the study may have been based on the patients' monocular subjective perception of vision rather than their overall level of eyesight or its impact on the quality of their life.

These data raise a series of important issues. The key issue, which is undoubtedly uncomfortable for patients, eye-care professionals, and policy makers, is that cataract surgery may still be effective at a monocular level in improving vision, even when the scope for quality of life improvement is limited. Those who receive healthcare, as a result of their preconceptions may request surgery in order to improve monocular vision. Those who supply healthcare may be pleased to provide surgery on request. However, policy makers involved in payment for healthcare would find it less expensive to set thresholds based on overall quality of life (using both eyes together). The most important question is whether surgery should be rationed according to overall visual functioning or whether monocular intervention should be encouraged in the absence of significant functional impairment. Reasonable decisions on 'demand management' could be possible if further, more detailed information were available regarding the 'risk-benefit' ratios of various threshold levels for surgery. It may then also be necessary to re-educate the public so that their expectations conform to identified principles of risk management as well as possible rationing of care away from those cataract patients whose overall vision and quality of life are only mildly affected. These results also raise the awkward question about the definition of 'patientcentred care'. The surgeons in the study may have acted in a patient-centred manner, fulfilling the wishes and expectations of their patients in the absence of clear risk-benefit information, which would include the expected quality of life gains (if any). These results indicate a need for further work to clarify risk-benefit issues at various thresholds for surgical intervention.

\section{References}

1 Desai P, Reidy A, Minassian DC. Profile of patients presenting for cataract surgery in the UK: national data collection. Br J Ophthalmol 1999; 83: 893-896.

2 Brown NAP. The morphology of cataract and visual performance. Eye 1993; 7: 63-67.

3 Frost NA, Sparrow JM. Use of vision tests in clinical decision making about cataract surgery: Results of a national survey. Br J Ophthalmo 2000; 84: 432-434.

4 Hart PM, Chakravarthy U, Stevenson MR. Questionnairebased survey on the importance of quality of life measures in practice. Eye 1998; 12: 124-126. 
5 Schein OD, Steinberg EP, Cassard SD, Tielsch JM, Sommer A. Predictors of outcome in patients who underwent cataract surgery. Ophthalmology. 1995; 102: 817-823.

6 Frost NA, Sparrow JM, Durrant JS, Donoval JL, Peters TJ, Brookes, ST. Development of a questionnaire for measurement of vision-related quality of life. Ophthal Epidemiol 1998; 5: 185-210.

7 Department of Health and Social Services National Statistics. Census Tables 2001. Hospital Episode Statistics, London.

8 Stenevi U, Lundstrom M, Thornburn $\mathrm{W}$. The cost of patients awaiting cataract surgery. Acta Ophthalmol Scand. 2000; 78: 703-705.

9 O'Day DM. Outcomes after cataract surgery (discussion). Ophthalmology 1994; 100: 1140-1141.

10 O'Day DM. Socioeconomics viewpoint: the need for an update of the clinical practice guideline on cataract [review]. Arch Ophthalmol 1995; 113: 718-720.

11 Sletteberg O, Hovding G, Bertelsen T. Do we operate too many cataracts? The referred cataract patients' own appraisal of their need for surgery. Acta Ophthalmol 1995; 73: 77-80.

12 Tobacman JK, Lee P, Zimmerman B, Kolder H, Hilborne L, Brook R. Assessment of appropriateness of cataract surgery at ten academic medical centers in 1990. Ophthalmology 1996; 103: 207-215.
13 Damiano AM, Steinberg EP, Cassard SD, Bass EB Diener-West M, Legro MW et al. Comparison of generic versus disease-specific measures of functional impairment in patients with cataract. Med Care 1995; 33: AS120-AS130.

14 Alonso J, Espallargues M, Andersen TF, Cassard SD, Dunn E, Bernth-Petersen P et al. International applicability of the VF-14: an index of visual function in patients with cataracts. Ophthalmology 1997; 104: 799-807.

15 Norregaard JC, Bernth-Petersen P, Andersen TF. Visual impairment and general health among Danish cataract patients. Results from the Danish Cataract Surgery Outcomes Study. I. Acta Ophthalmol 1996; 74: 598-603.

16 Mildon D, Noertjojo K, Rollins D, Blicker J, Law F, Courtright $\mathrm{P}$ et al. Cataract surgery at the Vancouver Eye Care Centre: do patient indications meet provincial clinical practice guidelines? Can J Ophthalmol. 2003; 38: 199-206.

17 Tobacman JK, Zimmerman B, Lee P, Hilborne L, Kolder H, Brook RH et al. Visual acuity following cataract surgeries in relationship to pre-operative appropriateness ratings. Med Decision Making 2003; 23: 122-130.

18 Desai P, Reidy A, Minassian DC, Vafidis G, Bolger J. Gains from cataract surgery: visual function and quality of life. $\mathrm{Br}$ J Ophthalmol 1996; 80: 868-873. 